\title{
Large multicystic ovaries in a young girl with severe hypothyroidism - an unusual association
}

\author{
Harpreet Kour Isher ${ }^{1}$, Gurmandeep Kaur ${ }^{1}$, Sukhwinder Bajwa ${ }^{1}$, Anita Singh ${ }^{1}$, Santprakash S Goraya ${ }^{1}$ \\ Sri Lanka Journal of Obstetrics and Gynaecology 2012; 34: 110-111
}

\section{Introduction}

Severe and occult hypothyroidism of long duration may infrequently present as large ovarian cysts. Hunter ${ }^{1}$ found the prevalance of hypothyroidism in young population $<22$ years to be $0.135 \%$, with a rising trend. An increased awareness of an unusual presentation of hypothyroidism as ovarian cysts in the younger female age group is thus, necessary. We are reporting a case of 21 year adult female who presented with large bilateral multicystic ovaries which resolved dramatically after thyroid replacement therapy.

\section{Case report}

Written consent was taken from the patient and the departmental ethical committee approved this report. A 21 year unmarried female presented with the chief complaints of menorrhagia for the last 6 months with pain abdomen on and off for the last one month. She had been bleeding heavily for the last 12 days, along with the passage of clots. Over the last 6 months her cycle interval had increased from 45 to 60 days and bleeding had increased in duration and amount. Her menarche was at a normal age of 13 years followed by regular cycles till the last 6 months. There was history of weight gain and malaise for last 2-3 yrs. Her physical examination revealed weight $73 \mathrm{~kg}$, height 5 feet and BMI 31.4 (obese category). She had apathetic expressions. Her pulse was $96 \mathrm{bpm}$ and BP 130/80 mm Hg. Her IQ was normal. She was pale with periorbital puffiness, dry skin and swelling over hands and feet and slow reflexes. Thyroid gland was not enlarged and there was no galactorrhea. Secondary sexual characters were normally developed. On abdominal examination, an abdominopelvic mass

\footnotetext{
${ }^{1}$ Department of Obstetrics and Gynaecology, Gian Sagar Medical College and Hospital, Ramnagar, Banur, Punjab, India.
}

Correspondence: Harpreet Kour Isher

E-mail: harpreetsukhija1@yahoo.com reaching upto the umbilicus was felt, which was cystic, non tender and mobile side to side. Per rectal examination revealed cystic masses with approximate dimensions of $10 \times 12 \mathrm{~cm}$ and $6 \times 6 \mathrm{~cm}$ in left and right fornix respectively, with uterus of normal size.

Her lab tests revealed mild anemia with a picture of dimorphic nature on peripheral blood film. Ultrasound abdomen and pelvis showed bilateral enlarged multicystic ovaries; right ovary $12 \times 9 \mathrm{~cm}$ and left ovary $5.9 \times 4.9 \mathrm{~cm}$. Uterus was normal with endometrial thickness of $6.5 \mathrm{~mm}$. Her CA 125 was $24.6 \mathrm{U} / \mathrm{ml}$ and beta HCG $<0.5 \mathrm{mIU} / \mathrm{ml}$. Lipid profile was deranged (hypertriglyceridemia) and liver function tests revealed a low alkaline phosphatase. Blood sugar was normal. Her TSH was markedly raised $>100 \mathrm{uIU} / \mathrm{ml}$, and $\mathrm{T} 3, \mathrm{~T} 40.6 \mathrm{ng} / \mathrm{ml}$ and $0.5 \mathrm{ug} /$ dl respectively. Ultrasound thyroid showed smaller thyroid lobes with heterogenous coarsened echo pattern suggestive of chronic thyroiditis. Antithyroid peroxidase was borderline positive $52.50 \mathrm{U} / \mathrm{ml}$ (positive $>50$ ) and antithyroglobulin antibodies were negative. Serum prolactin was raised at $68.6 \mathrm{ng} / \mathrm{ml}$ and serum FSH was normal. Chest and skull radiographs were normal with normal pituitary fossa. ECG showed sinus tachycardia.

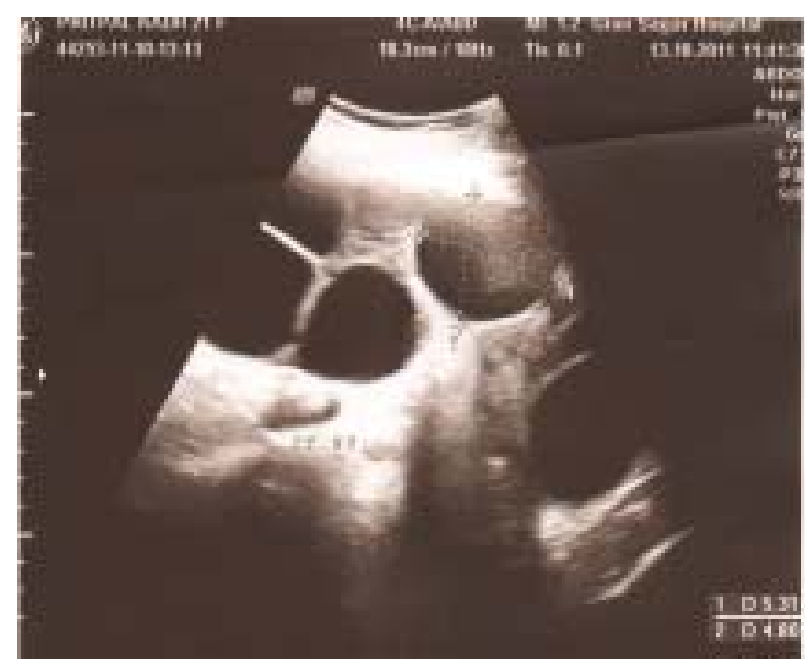

Figure 1. Sonogram showing left multicystic ovary 


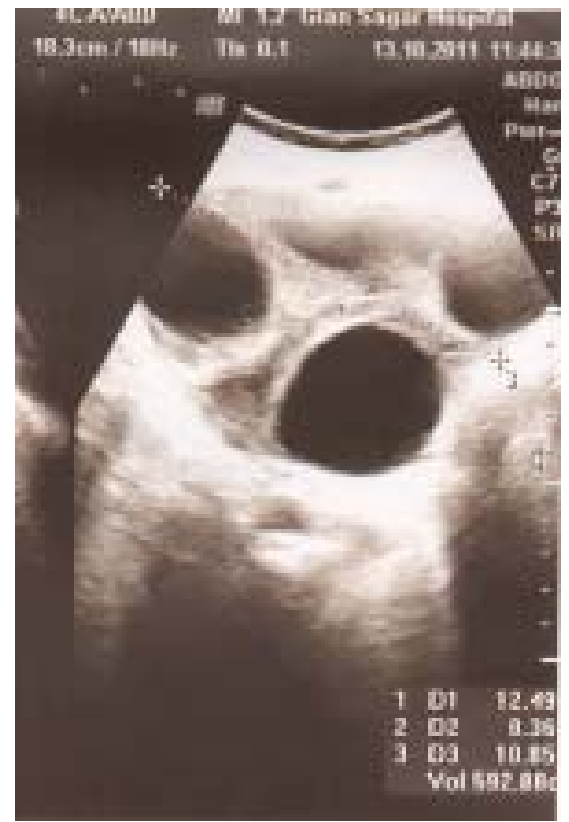

Figure 2. Sonogram showing right multicystic ovary.

Treatment and follow up: Our patient was started on $25 \mathrm{ug}$ of thyroxine and gradually increased to $100 \mathrm{ug}$. The acute bleeding episode was controlled with oral progesterone and hemostatic drugs. She was started on oral hematinics and calcium. By 6 weeks, both the ovarian cysts had resolved completely. At the 3 month follow up, patient was euthyroid with normal menstrual cycles and weighed $70 \mathrm{~kg}$.

\section{Discussion}

Primary hypothyroidism is a common problem. It can infrequently lead to cystic ovarian enlargement that may or may not be hormonally active. The ovarian enlargement could present as a large ovarian cyst, multicystic ovaries, polycystic ovaries or spontaneous hyperstimulation. The etiopathogenesis of ovarian cysts in hypothyroidism is complex. In 1960, Van Wyk and Grumbach first described a syndrome of breast enlargement, multicystic ovaries and uterine bleeding in association with long standing juvenile hypothyroidism ${ }^{2}$. They proposed that there was a 'hormonal overlap' in the pituitary feedback mechanism. The overlap is due to the fact that TSH, $\mathrm{GH}, \mathrm{FSH}$ and LH are all glycoprotein's with common alpha chains and may thus cross react. High TSH could produce FSH and LH-like activity leading to luteinized ovarian cysts ${ }^{3,4}$. The thyroid releasing hormone may also act on pituitary cells to stimulate gonadotropin release, and hence, FSH and LH. The pituitary feed-back due to high TSH could also lead to pituitary enlargement and adenomas as described in literature ${ }^{5}$. Increased ovarian sensitivity to gona- dotropins, altered metabolism of estrogens, hypothalamic pituitary dysfunction, altered prolactin metabolism, and interference of myxedematous infiltrate with steroidogenesis in ovary are the other postulated mechanisms ${ }^{6}$.

The diagnosis of ovarian cysts was a coincidental clinical finding in our case corroborated on ultrasound and the ovarian cysts may have been responsible for the abnormal uterine bleeding in our case. Histopathology of the ovarian cyst was not available as no surgical intervention was done. There was no pituitary enlargement though serum prolactin levels were raised. The ovarian cysts were the first to regress (within 6 weeks) followed by normalizing of serum prolactin and thyroid hormones sequentially.

Results with simple medical intervention as thyroxine replacement therapy are very rewarding as in our patient. Surgery in a patient with ovarian cyst of endocrine (hypothyroidism) origin is warranted only in cases of complications as torsion or rupture or if the cyst persists in spite of hormone therapy.

To conclude, even a large ovarian cyst $(>10 \mathrm{~cm})$ will not warrant the traditional approach of laparotomy ${ }^{7}$ if hypothyroidism is the cause. Awareness about this association can help avoid unnecessary investigation to rule out malignancy, especially in young girls. Besides, a pelvic sonogram in cases of severe hypothyroidism might also detect ovarian cysts incidentally.

\section{References}

1. Hunter I, Greene SA, MacDonald MT, et al. Prevalance and aeitiology of hypothyroidism in young. Arch Dis Child 2000; 83: 207-10.

2. Van Wyk JJ, Grumbach MM. Syndrome of precocious menstruation and galactorrhea in juvenile hypothyroidism: An example of hormonal overlap in pituitary feedback. J Pediatr 1960; 57: 416-35.

3. Evers JLH, Rolland R. Primary hypothyroidism and ovarian activity: evidence for overlap in the synthesis of pituitary glycoproteins. Case report. Br J Obstet Gynaecol 1981; 88: 195-202.

4. Anasti JN, FlackMR, Froehlich, et al. A potential novel mechanism for precocious puberty in juvenile hypothyroidism. J Clin Endocrinol Metab 1995; 80: 276-9.

5. Yam ashita Y, Kawamura T, Fujikawa R, et al. Regression of both pituitary and ovarian cysts after administration of thyroid hormone in a case of primary hypothyroidism. Intern Med 2001; 40: 751-5.

6. Hansen K,Tho S, Hanly M, et al. Massive ovarian enlargement in primary hypothyroidism. Fertil Steril 1997; 67: 169-71.

7. Graham L. ACOG Practice Guidelines on Management of Adnexal Masses. Am Fam Physician 2008; 77(9): 1320-3. 